\title{
COMPACT COMPOSITION OPERATORS ON THE BLOCH SPACE
}

\author{
KEVIN MADIGAN AND ALEC MATHESON
}

\begin{abstract}
Necessary and sufficient conditions are given for a composition operator $C_{\phi} f=f \circ \phi$ to be compact on the Bloch space $\mathscr{B}$ and on the little Bloch space $\mathscr{B}_{0}$. Weakly compact composition operators on $\mathscr{B}_{0}$ are shown to be compact. If $\phi \in \mathscr{B}_{0}$ is a conformal mapping of the unit disk $\mathbb{D}$ into itself whose image $\phi(\mathbb{D})$ approaches the unit circle $\mathbb{T}$ only in a finite number of nontangential cusps, then $C_{\phi}$ is compact on $\mathscr{B}_{0}$. On the other hand if there is a point of $\mathbb{T} \cap \overline{\phi(\mathbb{D})}$ at which $\phi(\mathbb{D})$ does not have a cusp, then $C_{\phi}$ is not compact.
\end{abstract}

\section{INTRODUCTION}

Let $\mathbb{D}$ denote the unit disk in the complex plane. A function $f$ holomorphic in $\mathbb{D}$ is said to belong to the Bloch space $\mathscr{B}$ if

$$
\sup _{z \in \mathbf{D}}\left(1-|z|^{2}\right)\left|f^{\prime}(z)\right|<\infty
$$

and to the little Bloch space $\mathscr{B}_{0}$ if

$$
\lim _{|z| \rightarrow 1}\left(1-|z|^{2}\right)\left|f^{\prime}(z)\right|=0 \text {. }
$$

It is well known that $\mathscr{B}$ is a Banach space under the norm

$$
\|f\|_{\mathscr{B}}=|f(0)|+\sup _{z \in \mathbb{D}}\left(1-|z|^{2}\right)\left|f^{\prime}(z)\right|
$$

and that $\mathscr{B}$ is a closed subspace of $\mathscr{B}$. Furthermore, $\mathscr{B}$ is isometrically isomorphic to the second dual of $\mathscr{B}_{0}$ and the inclusion $\mathscr{B}_{0} \subset \mathscr{B}$ corresponds to the canonical imbedding of $\mathscr{B}_{0}$ into $\mathscr{B}_{0}^{* *}$ [ACP]. It is a simple consequence of the Schwarz-Pick lemma [A] that a holomorphic mapping $\phi$ of the unit disk into itself induces a bounded composition operator $C_{\phi} f=f \circ \phi$ on $\mathscr{B}$. Indeed, if $f \in \mathscr{B}$, then

$$
\begin{aligned}
\left(1-|z|^{2}\right)\left|(f \circ \phi)^{\prime}(z)\right| & =\left(1-|z|^{2}\right)\left|f^{\prime}(\phi(z))\right|\left|\phi^{\prime}(z)\right| \\
& =\frac{1-|z|^{2}}{1-|\phi(z)|^{2}}\left|\phi^{\prime}(z)\right|\left(1-|\phi(z)|^{2}\right)\left|f^{\prime}(\phi(z))\right|
\end{aligned}
$$

Received by the editors November 23, 1993 and, in revised form, April 27, 1994; originally communicated to the Proceedings of the AMS by Theodore Gamelin.

1991 Mathematics Subject Classification. Primary 47B38, 47B07; Secondary 30D55.

Key words and phrases. Composition operator, compact operator, Bloch space, cusp.

The authors would like to thank Dan Luecking for bringing Proposition 1 to their attention and David Stegenga for discussions about cusps. 
and the Schwarz-Pick lemma guarantees that

$$
\frac{1-|z|^{2}}{1-|\phi(z)|^{2}}\left|\phi^{\prime}(z)\right| \leq 1 .
$$

Since the identity function $f(z)=z$ belongs to $\mathscr{B}_{0}$, it is clear that $\phi \in \mathscr{B}_{0}$ if $C_{\phi}$ maps $\mathscr{B}_{0}$ into itself. Conversely, if $\phi \in \mathscr{B}_{0}$ and $f \in \mathscr{B}_{0}$, it follows from (1) and (2) that $f \circ \phi \in \mathscr{B}_{0}$. Indeed, if $\epsilon>0$, there exists $\delta>0$ such that $\left(1-|z|^{2}\right)\left|f^{\prime}(z)\right|<\epsilon$ whenever $|z|^{2}>1-\delta$. In particular, $\left(1-|z|^{2}\right)\left|(f \circ \phi)^{\prime}(z)\right|<$ $\epsilon$ whenever $|\phi(z)|^{2}>1-\delta$. On the other hand, if $|\phi(z)|^{2} \leq 1-\delta$,

$$
\left(1-|z|^{2}\right)\left|(f \circ \phi)^{\prime}(z)\right| \leq \frac{\|f\|_{\mathscr{B}}}{\delta}\left(1-|z|^{2}\right)\left|\phi^{\prime}(z)\right|,
$$

and the right-hand side tends to 0 as $|z| \rightarrow 1$.

In Section 2 the compact composition operators on $\mathscr{B}_{0}$ and on $\mathscr{B}$ will be characterized in terms of the quotient $\frac{1-|z|^{2}}{1-|\phi(z)|^{2}}\left|\phi^{\prime}(z)\right|$. A bounded linear operator $T: X \rightarrow Y$ from the Banach space $X$ to the Banach space $Y$ is weakly compact if $T$ takes bounded sets in $X$ into relatively weakly compact sets in $Y$. Gantmacher's theorem [D, p. 21] asserts that $T$ is weakly compact if and only if $T^{* *}\left(X^{* *}\right) \subset Y$ where $T^{* *}$ denotes the second adjoint of $T$. This theorem and the characterization of compact operators on $\mathscr{B}_{0}$ will be used to show that every weakly compact composition operator on $\mathscr{B}_{0}$ is compact.

In Section 3 the results of Section 2 will be applied to certain univalent functions $\phi$ which map $\mathbb{D}$ into itself. It is known that such functions belong to $\mathscr{B}_{0}\left[\mathrm{P}\right.$, p. 12]; and it will be clear from Section 2 that if $\|\phi\|_{\infty}<1$, then $C_{\phi}$ is compact on $\mathscr{B}_{0}$. On the other hand if $\|\phi\|_{\infty}=1$ and there is a point of $\mathbb{T} \cap \overline{\phi(\mathbb{D})}$ at which $\phi(\mathbb{D})$ does not have a cusp, then $C_{\phi}$ is not compact. However if $\mathbb{T} \cap \overline{\phi(\mathbb{D})}$ consists of only one point at which $\phi(\mathbb{D})$ has a nontangential cusp, then $C_{\phi}$ is compact on $\mathscr{B}_{0}$.

\section{COMPACTNESS}

Theorem 1 gives a precise description of those $\phi$ which induce compact composition operators on $\mathscr{B}_{0}$. It will be useful first to give a criterion for compactness in $\mathscr{B}_{0}$.

Lemma 1. A closed set $K$ in $\mathscr{B}_{0}$ is compact if and only if it is bounded and satisfies

$$
\lim _{|z| \rightarrow 1} \sup _{f \in K}\left(1-|z|^{2}\right)\left|f^{\prime}(z)\right|=0 .
$$

Proof. First suppose that $K$ is compact and let $\epsilon>0$. Choose an $\epsilon / 2$-net $f_{1}$, $f_{2}, \ldots, f_{n}$ in $K$. There is an $r, 0<r<1$, such that $\left(1-|z|^{2}\right)\left|f_{i}^{\prime}(z)\right|<\epsilon / 2$ if $|z|>r, 1 \leq i \leq n$. If $f \in K,\left\|f-f_{i}\right\|_{\mathscr{B}}<\epsilon / 2$ for some $f_{i}$ and so

$$
\left(1-|z|^{2}\right)\left|f^{\prime}(z)\right| \leq\left\|f-f_{i}\right\|_{\mathscr{B}}+\left(1-|z|^{2}\right)\left|f_{i}^{\prime}(z)\right|<\epsilon
$$

whenever $|z|>r$. This establishes (3).

On the other hand if $K$ is a closed bounded set which satisfies $(3)$ and $\left(f_{n}\right)$ is a sequence in $K$, then by Montel's theorem there is a subsequence $\left(f_{n_{k}}\right)$ which converges uniformly on compact subsets of $\mathbb{D}$ to some holomorphic function $f$. Then also $\left(f_{n_{k}}^{\prime}\right)$ converges uniformly to $f^{\prime}$ on compact subsets of $\mathbb{D}$. By (3), if 
$\epsilon>0$, there is an $r, 0<r<1$, such that for all $g \in K,\left(1-|z|^{2}\right)\left|g^{\prime}(z)\right|<\epsilon / 2$ if $|z|>r$. It follows that $\left(1-|z|^{2}\right)\left|f^{\prime}(z)\right|<\epsilon / 2$ if $|z|>r$. Since $\left(f_{n_{k}}\right)$ converges uniformly to $f$ and $\left(f_{n_{k}}^{\prime}\right)$ converges uniformly to $f^{\prime}$ on $|z| \leq r$, it follows that $\lim \sup _{k \rightarrow \infty}\left\|f_{n_{k}}-f\right\|_{\mathscr{B}} \leq \epsilon$. Since $\epsilon>0, \lim _{k \rightarrow \infty}\left\|f_{n_{k}}-f\right\|_{\mathscr{B}}=0$ and so $K$ is compact.

Theorem 1. If $\phi$ is a holomorphic mapping of the unit disk $\mathbb{D}$ into itself, then $\phi$ induces a compact composition operator on $\mathscr{B}_{0}$ if and only if

$$
\lim _{|z| \rightarrow 1} \frac{1-|z|^{2}}{1-|\phi(z)|^{2}}\left|\phi^{\prime}(z)\right|=0 .
$$

Proof. It follows from Lemma 1 that $C_{\phi}$ is compact on $\mathscr{B}_{0}$ if and only if

$$
\lim _{|z| \rightarrow 1} \sup _{\|f\|_{\mathscr{\theta}} \leq 1}\left(1-|z|^{2}\right)\left|(f \circ \phi)^{\prime}(z)\right|=0 .
$$

But

$$
\left(1-|z|^{2}\right)\left|(f \circ \phi)^{\prime}(z)\right|=\frac{1-|z|^{2}}{1-|\phi(z)|^{2}}\left|\phi^{\prime}(z)\right|\left(1-|\phi(z)|^{2}\right)\left|f^{\prime}(\phi(z))\right|,
$$

and

$$
\sup _{\|f\|_{\infty} \leq 1}\left(1-|w|^{2}\right)\left|f^{\prime}(w)\right|=1
$$

for each $w \in \mathbb{D}$. The theorem follows.

It should be remarked that (4) implies $\phi \in \mathscr{B}_{0}$. A similar condition characterizes compact composition operators on $\mathscr{B}$.

Theorem 2. If $\phi$ is a holomorphic mapping of the unit disk $\mathbb{D}$ into itself, then $\phi$ induces a compact composition operator on $\mathscr{B}$ if and only if for every $\epsilon>0$, there exists $r, 0<r<1$, such that

$$
\frac{1-|z|^{2}}{1-|\phi(z)|^{2}}\left|\phi^{\prime}(z)\right|<\epsilon
$$

whenever $|\phi(z)|>r$.

Proof. First assume that (5) holds. In order to prove that $C_{\phi}$ is compact on $\mathscr{B}$ it is enough to show that if $\left(f_{n}\right)$ is a bounded sequence in $\mathscr{B}$ which converges to 0 uniformly on compact subsets of $\mathbb{D}$, then $\left\|f_{n} \circ \phi\right\|_{\mathscr{B}} \rightarrow 0$. Let $M=$ $\sup _{n}\left\|f_{n}\right\|_{\mathscr{B}}$. Given $\epsilon>0$ there exists $r, 0<r<1$, such that $\frac{1-|z|^{2}}{1-|\phi(z)|^{2}}\left|\phi^{\prime}(z)\right|<$ $\frac{\epsilon}{2 M}$ if $|\phi(z)|>r$. Since

$$
\begin{aligned}
\left(1-|z|^{2}\right)\left|\left(f_{n} \circ \phi\right)^{\prime}(z)\right| & =\frac{1-|z|^{2}}{1-|\phi(z)|^{2}}\left|\phi^{\prime}(z)\right|\left(1-|\phi(z)|^{2}\right)\left|f_{n}^{\prime}(\phi(z))\right| \\
& \leq M \frac{1-|z|^{2}}{1-|\phi(z)|^{2}}\left|\phi^{\prime}(z)\right|,
\end{aligned}
$$

it follows that $\left(1-|z|^{2}\right)\left|\left(f_{n} \circ \phi\right)^{\prime}(z)\right|<\frac{\epsilon}{2}$ if $|\phi(z)|>r$. On the other hand, $f_{n} \circ \phi(0) \rightarrow 0$ and $\left(1-|w|^{2}\right)\left|f_{n}^{\prime}(w)\right| \rightarrow 0$ uniformly for $|w| \leq r$. Since

$$
\left(1-|z|^{2}\right)\left|\left(f_{n} \circ \phi\right)^{\prime}(z)\right| \leq\left(1-|\phi(z)|^{2}\right)\left|f_{n}^{\prime}(\phi(z))\right|,
$$

it follows that for largc enough $n,\left|f_{n} \circ \phi(0)\right|<\frac{\epsilon}{2}$ and $\left(1-|z|^{2}\right)\left|\left(f_{n} \circ \phi\right)^{\prime}(z)\right|<\frac{\epsilon}{2}$ if $|\phi(z)| \leq r$. Hence $\left\|f_{n} \circ \phi\right\|_{\mathscr{B}}<\epsilon$ for large $n$. 
Now assume that (5) fails. Then there exists a subsequence $\left(z_{n}\right)$ in $\mathbb{D}$ and an $\epsilon>0$ such that $\left|z_{n}\right| \rightarrow 1$ and $\frac{1-\left|z_{n}\right|^{2}}{1-\left|\phi\left(z_{n}\right)\right|^{2}}\left|\phi^{\prime}\left(z_{n}\right)\right|>\epsilon$ for all $n$. Passing to a subsequence if necessary it may be assumed that $w_{n}=\phi\left(z_{n}\right) \rightarrow w_{0} \in \mathbb{T}$. Let $f_{n}(z)=\log \frac{1}{1-\bar{w}_{n} z}$. Then $\left(f_{n}\right)$ converges to $f_{0}$ uniformly on compact subsets of $\mathbb{D}$. On the other hand,

$$
\begin{aligned}
\left\|C_{\phi} f_{n}-C_{\phi} f_{0}\right\|_{\mathscr{B}} & \geq\left(1-\left|z_{n}\right|^{2}\right)\left|\left(C_{\phi} f_{n}\right)^{\prime}\left(z_{n}\right)-\left(C_{\phi} f_{0}\right)^{\prime}\left(z_{n}\right)\right| \\
& =\left(1-\left|z_{n}\right|^{2}\right)\left|\phi^{\prime}\left(z_{n}\right)\right|\left|\frac{\bar{w}_{n}}{1-\left|w_{n}\right|^{2}}-\frac{\bar{w}_{0}}{1-\bar{w}_{0} w_{n}}\right| \\
& =\frac{\left(1-\left|z_{n}\right|^{2}\right)}{1-\left|w_{n}\right|^{2}}\left|\phi^{\prime}\left(z_{n}\right)\right|\left|\frac{\bar{w}_{n}-\bar{w}_{0}}{1-\bar{w}_{0} w_{n}}\right| \\
& >\epsilon
\end{aligned}
$$

for all $n$, so $C_{\phi} f_{n}$ does not converge to $C_{\phi} f_{0}$ in norm. Hence $C_{\phi}$ is not compact.

It is important to note that although (4) implies (5), since in this case $C_{\phi}$ on $\mathscr{B}$ is the second adjoint of $C_{\phi}$ on $\mathscr{B}_{0}$, the two conditions are not equivalent. Condition (4) implies that $\phi \in \mathscr{B}_{0}$, while there certainly exist functions $\phi \notin$ $\mathscr{B}_{0}$ which satisfy (5). Indeed, any $\phi$ for which $\|\phi\|_{\infty}<1$ satisfies (5) trivially.

A sequence $\left(w_{n}\right)$ in $\mathbb{D}$ is said to be $\eta$-separated if $\rho\left(w_{n}, w_{m}\right)=\left|\frac{w_{m}-w_{n}}{1-\bar{w}_{m} w_{n}}\right|>$ $\eta$ whenever $m \neq n$. Thus an $\eta$-separated sequence consists of points which are uniformly far apart in the pseudohyperbolic metric on $\mathbb{D}$, or equivalently, the hyperbolic balls $\Delta\left(w_{n}, r\right)=\left\{z \mid \rho\left(z, w_{n}\right)<r\right\}$ are pairwise disjoint for some $r>0$. Evidently any sequence $\left(w_{n}\right)$ in $\mathbb{D}$ which satisfies $\left|w_{n}\right| \rightarrow 1$ possesses an $\eta$-separated subsequence for any $\eta>0$. In particular, if the sequence $\left(w_{n}\right)$ in the proof of Theorem 2 is $\eta$-separated, then the calculation in the proof shows that $\left\|C_{\phi} f_{m}-C_{\phi} f_{n}\right\|>\epsilon \eta$ whenever $m \neq n$, so $\left(C_{\phi} f_{n}\right)$ has no norm convergent subsequences.

Another property of separated sequences is contained in the next proposition. This proposition is related to some interpolation results of Rochberg [RR 1 , RR2]. Since the method of proof is precisely the same as Rochberg's, a proof will only be sketched.

Proposition 1. There is an absolute constant $R>0$ such that if $\left(w_{n}\right)$ is $R$ separated, then for every bounded sequence $\left(\lambda_{n}\right)$ there is an $f \in \mathscr{B}$ such that $\left(1-\left|w_{n}\right|^{2}\right) f^{\prime}\left(w_{n}\right)=\lambda_{n}$ for all $n$.

The idea of the proof is to consider two operators $S: \mathscr{B} \rightarrow l^{\infty}$ given by

$$
S(f)_{n}=\left(1-\left|w_{n}\right|^{2}\right) f^{\prime}\left(w_{n}\right)
$$

and $T: l^{\infty} \rightarrow \mathscr{B}$ given by

$$
T(\lambda)(z)=\sum_{n=1}^{\infty} \lambda_{n} \frac{1}{3 \bar{w}_{n}} \frac{\left(1-\left|w_{n}\right|^{2}\right)^{3}}{\left(1-\bar{w}_{n} z\right)^{3}}
$$

where $\lambda=\left(\lambda_{n}\right) \in l^{\infty}$. The proposition will follow if it can be shown that $\|I-S T\|<1$, for then $S T$ will be invertible and so $S$ will be onto. The symbol $C$ will denote a constant whose value changes from place to place but 
does not depend on $R$. Now

$$
(S T-I)(\lambda)_{n}=\left(1-\left|w_{n}\right|^{2}\right) \sum_{m \neq n} \lambda_{m} \frac{\left(1-\left|w_{m}\right|^{2}\right)^{3}}{\left(1-\bar{w}_{m} w_{n}\right)^{4}}
$$

and so it will be enough to estimate

$$
\sup _{n}\left(1-\left|w_{n}\right|\right) \sum_{m \neq n} \frac{\left(1-\left|w_{m}\right|^{2}\right)^{3}}{\left|1-\bar{w}_{m} w_{n}\right|^{4}}
$$

If $R>\mathrm{i} / 2$, say, then there is a fixed $\delta>0$ such that the Euclidean disk $D_{m}$ of center $w_{m}$ and radius $\delta\left(1-\left|w_{m}\right|^{2}\right)$ is contained in the hyperbolic disk $\Delta_{m}=\Delta\left(w_{m}, R\right)$ and is disjoint from the hyperbolic disks $\Delta_{n}$ for $n \neq m$. Since $\left|1-\bar{z} w_{n}\right|^{-4}$ is subharmonic and the radius of $D_{m}$ is comparable to $1-\left|w_{m}\right|^{2}$,

$$
\frac{\left(1-\left|w_{m}\right|^{2}\right)^{3}}{\left|1-\bar{w}_{m} w_{n}\right|^{4}} \leq C \iint_{D_{m}} \frac{1-\left|w_{m}\right|^{2}}{\left|1-\bar{z} w_{n}\right|^{4}} d x d y
$$

and since $\left|1-\bar{w}_{n} z\right|$ dominates $1-\left|w_{m}\right|^{2}$ on $D_{m}$, it follows that

$$
\frac{\left(1-\left|w_{m}\right|^{2}\right)^{3}}{\left|1-\bar{w}_{m} w_{n}\right|^{4}} \leq C \iint_{D_{m}} \frac{1}{\left|1-\bar{w}_{n} z\right|^{3}} d x d y
$$

and hence

$$
\begin{aligned}
\sup _{n}\left(1-\left|w_{n}\right|\right) \sum_{m \neq n} \frac{\left(1-\left|w_{m}\right|^{2}\right)^{3}}{\left|1-\bar{w}_{m} w_{n}\right|^{4}} & \leq C \iint_{\cup_{m \neq n} D_{m}} \frac{1-\left|w_{n}\right|^{2}}{\left|1-\bar{w}_{n} z\right|^{3}} d x d y \\
& \leq C \iint_{\mathbb{D} \backslash \Delta_{n}} \frac{1-\left|w_{n}\right|^{2}}{\left|1-\bar{w}_{n} z\right|^{3}} d x d y .
\end{aligned}
$$

The change of variables $z=\frac{w_{n}+\zeta}{1+\bar{w}_{n} \zeta}$ turns this into

$$
\sup _{n}\left(1-\left|w_{n}\right|\right) \sum_{m \neq n} \frac{\left(1-\left|w_{m}\right|^{2}\right)^{3}}{\left|1-\bar{w}_{m} w_{n}\right|^{4}} \leq C \iint_{|\zeta|>R} \frac{1}{\left|1+\bar{w}_{n} \zeta\right|} d \xi d \eta,
$$

and the last integral can be made arbitrarily small uniformly in $n$ if $R$ is chosen close enough to 1 . This provides the desired estimate.

Since every sequence $\left(w_{n}\right)$ with $\left|w_{n}\right| \rightarrow 1$ contains an $R$-separated subsequence $\left(w_{n_{k}}\right)$, it follows that there is an $f \in \mathscr{B}$ such that $\left(1-\left|w_{n_{k}}\right|^{2}\right) f^{\prime}\left(w_{n_{k}}\right)=$ 1 for all $k$. This will be used in the proof of the next theorem.

Theorem 3. Every weakly compact composition operator $C_{\phi}$ on $\mathscr{B}_{0}$ is compact. Proof. The composition operator $C_{\phi}: \mathscr{B}_{0} \rightarrow \mathscr{B}_{0}$ is compact if and only if

$$
\lim _{|z| \rightarrow 1} \frac{1-|z|^{2}}{1-|\phi(z)|^{2}}\left|\phi^{\prime}(z)\right|=0
$$

and, according to Gantmacher's theorem, weakly compact if and only if $C_{\phi} f \in$ $\mathscr{B}_{0}$ for every $f \in \mathscr{B}$. If $C_{\phi}$ is not compact, there is an $\epsilon>0$ and a sequence $\left(z_{n}\right),\left|z_{n}\right| \rightarrow 1$, such that

$$
\frac{1-\left|z_{n}\right|^{2}}{1-\left|\phi\left(z_{n}\right)\right|^{2}}\left|\phi^{\prime}\left(z_{n}\right)\right| \geq \epsilon
$$


for all $n$. Since $\phi \in \mathscr{B}_{0},\left|\phi\left(z_{n}\right)\right| \rightarrow 1$, and by passing to a subsequence it may be assumed that $\left(\phi\left(z_{n}\right)\right)$ is $R$-separated. If $f \in \mathscr{B}$,

$$
\begin{aligned}
\left(1-\left|z_{n}\right|^{2}\right)\left|\left(C_{\phi} f\right)^{\prime}\left(z_{n}\right)\right| & =\frac{1-\left|z_{n}\right|^{2}}{1-\left|\phi\left(z_{n}\right)\right|^{2}}\left|\phi^{\prime}\left(z_{n}\right)\right|\left(1-\left|\phi\left(z_{n}\right)\right|^{2}\right)\left|f^{\prime}\left(\phi\left(z_{n}\right)\right)\right| \\
& \geq \epsilon\left(1-\left|\phi\left(z_{n}\right)\right|^{2}\right)\left|f^{\prime}\left(\phi\left(z_{n}\right)\right)\right| .
\end{aligned}
$$

Since $\left(\phi\left(z_{n}\right)\right)$ is $R$-separated, an application of Proposition 1 produces an $f \in \mathscr{B}$ such that $\left(1-\left|\phi\left(z_{n}\right)\right|^{2}\right)\left|\left(C_{\phi} f\right)^{\prime}\left(z_{n}\right)\right|=1$, for all $n$. Since $(1-$ $\left.\left|z_{n}\right|^{2}\right)\left|\left(C_{\phi} f\right)^{\prime}\left(z_{n}\right)\right| \geq \epsilon$ and $\left|z_{n}\right| \rightarrow 1, C_{\phi} f \notin \mathscr{B}_{0}$ and so $C_{\phi}$ is not weakly compact.

A slight refinement of these arguments will show that a noncompact composition operator on $\mathscr{B}_{0}$ must be an isomorphism on a subspace isomorphic to the sequence space $c_{0}$. This is not surprising since $\mathscr{B}_{0}$ is known to be isomorphic to $c_{0}$.

\section{EXAMPLES}

As remarked in the introduction any holomorphic mapping $\phi$ of the unit disk into itself satisfying $\|\phi\|_{\infty}<1$ induces a compact composition operator on $\mathscr{B}$ and also on $\mathscr{B}_{0}$ if $\phi \in \mathscr{B}_{0}$. On the other hand it is easy to see that if $\phi$ has a finite angular derivative at some point of $\mathbb{T}$, then $C_{\phi}$ cannot be compact. Indeed, $\phi$ has an angular derivative at $\zeta \in \mathbb{T}$ if the nontangential limit $\omega=f(\zeta) \in \mathbb{T}$ exists and if the quotient $\frac{f(z)-f(\zeta)}{z-\zeta}$ converges to some complex number $\mu$ as $z \rightarrow \zeta$ nontangentially. It is known that $\mu \neq 0$, and the Julia-Carathéodory lemma shows that $\frac{1-|z|^{2}}{1-|\phi(z)|^{2}}\left|\phi^{\prime}(z)\right|$ converges to $\zeta \bar{\omega} \mu \neq 0$ nontangentially. Applying Theorem 1 or 2 as appropriate shows that $C_{\phi}$ is not compact

It turns out, however, that $\phi$ can push the disk much more sharply into itself and still induce a noncompact composition operator. The easiest way to see this is to consider the functions $\phi_{\lambda, \alpha}(z)=1-\lambda(1-z)^{\alpha}, 0<\lambda, \alpha<1$. It is easy to see that $\phi_{\lambda, \alpha} \in \mathscr{B}_{0}$ and that $\phi_{\lambda, \alpha}$ maps $\mathbb{D}$ onto a region which behaves at 1 like a Stolz angle of opening $\pi \alpha$. If $C_{\phi}$ were compact on $\mathscr{B}_{0}$, composition with $\log \frac{1}{1-z}$ would yield a function in $\mathscr{B}_{0}$, but an easy calculation shows that this is not so. This leads to the consideration of cusps.

Throughout the remainder of this section $\phi$ will denote a univalent mapping of the unit disk $\mathbb{D}$ into itself with image $G=\phi(\mathbb{D})$. For simplicity it will be assumed that $\bar{G} \cap \mathbb{T}=\{1\}$. The region $G$ is said to have a cusp at $1[\mathrm{P}, \mathrm{p}$. 256] if

$$
\operatorname{dist}(w, \partial G)=o(|1-w|)
$$

as $w \rightarrow 1$ in $G$. Otherwise $G$ does not have a cusp at 1 . The cusp is said to be nontangential if $G$ lies inside a Stolz angle near 1, i.e., there exist $r, M>0$ such that

$$
|1-w| \leq M\left(1-|w|^{2}\right)
$$

if $|1-w|<r, w \in G$. Finally the following geometric property of the conformal mapping $\phi$ will be needed. If $\phi$ is a conformal mapping with domain $\mathbb{D}$, 
then

$$
\frac{1}{4}\left(1-|z|^{2}\right)\left|\phi^{\prime}(z)\right| \leq \operatorname{dist}(\phi(z), \partial G) \leq\left(1-|z|^{2}\right)\left|\phi^{\prime}(z)\right| .
$$

This inequality, known as the Koebe distortion theorem, is an elementary consequence of the Schwarz lemma and Koebe's one-quarter theorem [G, p. 13]. It can be used to prove that bounded univalent functions lie in $\mathscr{B}_{0}$. Indeed, if $\phi \notin \mathscr{B}_{0}$, there is a $\delta>0$ and a sequence $\left(z_{n}\right)$ in $\mathbb{D}$ with $\left|z_{n}\right| \rightarrow 1$ and $\left(1-\left|z_{n}\right|\right)\left|\phi^{\prime}\left(z_{n}\right)\right|>\delta$ for all $n$. Hence $\operatorname{dist}\left(\phi\left(z_{n}\right), \partial G\right)>\delta / 4$, so $\phi\left(z_{n}\right)$ has a cluster point in $G$, contradicting the fact that $\phi$ is a proper map. Theorem 4 provides a negative result.

Theorem 4. If $\phi$ is univalent and $G=\phi(\mathbb{D})$ satisfies $\bar{G} \cap \mathbb{T}=\{1\}$ but does not have a cusp at 1 , then $C_{\phi}$ is not compact on $\mathscr{B}_{0}$.

Proof. Since $G$ does not have a cusp at 1 , (6) fails. Hence there is a $\delta>0$ and a sequence $\left(z_{n}\right)$ in $\mathbb{D}$ such that $\left|z_{n}\right| \rightarrow 1$, but

$$
\operatorname{dist}\left(\phi\left(z_{n}\right), \partial G\right) \geq \delta\left|1-\phi\left(z_{n}\right)\right| \text {. }
$$

Hence

$$
\delta\left(1-\left|\phi\left(z_{n}\right)\right|^{2}\right) \leq 2 \delta\left(1-\left|\phi\left(z_{n}\right)\right|\right) \leq 2 \operatorname{dist}\left(\phi\left(z_{n}\right), \partial G\right) \leq 2\left(1-\left|z_{n}\right|^{2}\right)\left|\phi^{\prime}\left(z_{n}\right)\right|,
$$

so $\frac{1-\left|z_{n}\right|^{2}}{1-\left|\phi\left(z_{n}\right)\right|^{2}}\left|\phi^{\prime}\left(z_{n}\right)\right| \geq \frac{\delta}{2}$. Since $\left|z_{n}\right| \rightarrow 1$, Theorem 1 shows that $C_{\phi}$ is not compact.

The next theorem shows how to produce compact composition operators on $\mathscr{B}_{0}$ from univalent mappings $\phi$ with $\|\phi\|_{\infty}=1$.

Theorem 5. If $\phi$ is univalent and if $G$ has a nontangential cusp at 1 and touches the unit circle at no other point, then $C_{\phi}$ is a compact operator on $\mathscr{B}_{0}$.

Proof. As $\phi \in \mathscr{B}_{0}$, it will be enough to show that

$$
\lim _{|z| \rightarrow 1} \frac{1-|z|^{2}}{1-|\phi(z)|^{2}}\left|\phi^{\prime}(z)\right|=0,
$$

since the theorem will then follow from Theorem 1 . Since $G$ has a nontangential cusp at 1 , there exist $r, M>0$ such that

$$
|1-w| \leq M\left(1-|w|^{2}\right)
$$

if $|1-w|<r, w \in G$. Let $\epsilon>0$. Since $G$ has a cusp at 1 , there is a $\delta>0$ such that

$$
\operatorname{dist}(w, \partial G) \leq \frac{\epsilon}{4 M}|1-w|
$$

if $|1-w|<\delta, w \in G$. Let $\eta=\min (\delta, r)$. If $|1-\phi(z)|<\eta$, it follows that

$$
\begin{aligned}
\frac{1-|z|^{2}}{1-|\phi(z)|^{2}}\left|\phi^{\prime}(z)\right| & \leq \frac{4 \operatorname{dist}(\phi(z), \partial G)}{1-|\phi(z)|^{2}} \\
& \leq \frac{\epsilon}{M} \frac{|1-\phi(z)|}{1-|\phi(z)|^{2}} \\
& <\epsilon .
\end{aligned}
$$


On the other hand if $|1-\phi(z)| \geq \eta$, there is a constant $N>0$ such that $\left|\phi^{\prime}(z)\right| \leq N$ by the smoothness assumption and a $\rho>0$ such that $1-|\phi(z)|^{2} \geq$ $\rho$. In this case

$$
\frac{1-|z|^{2}}{1-|\phi(z)|^{2}}\left|\phi^{\prime}(z)\right| \leq \frac{N}{\rho}\left(1-|z|^{2}\right),
$$

and this is less than $\epsilon$ if $|z|^{2}>1-\frac{\rho \epsilon}{N}$. That completes the proof.

It is possible to describe regions $G$ with tangential cusp such that the Riemann mapping $\phi: \mathbb{D} \rightarrow G$ admits either possibility. Indeed, suppose that $h(\theta)$ and $k(\theta)$ are positive continuous functions on $\left[0, \theta_{0}\right]$ with $h(\theta)=o(\theta)$ and $k(\theta)=o(\theta)$. Let

$$
G=\left\{r e^{i \theta} \mid 0<\theta<\theta_{0}, h(\theta)<1-r<h(\theta)+k(\theta)\right\} .
$$

Then clearly $G$ has a tangential cusp at 1 . If $k(\theta)=o(h(\theta))$, then, for $w=$ $r e^{i \theta}=\phi(z)$,

$$
\left(1-|z|^{2}\right)\left|\phi^{\prime}(z)\right| \leq \operatorname{dist}(w, \partial G) \leq k(\theta)
$$

and

$$
1-|w|^{2} \geq 1-|w|>h(\theta),
$$

so $\frac{1-|z|^{2}}{1-|\phi(z)|^{2}}\left|\phi^{\prime}(z)\right| \rightarrow 0$ as $|\phi(z)| \rightarrow 1$. Since $\phi$ is univalent, the argument of Theorem 5 shows that $C_{\phi}$ is compact. On the other hand if $k(\theta)=2 h(\theta)$ and $w(\theta)=(1-2 h(\theta)) e^{i \theta}=\phi(z(\theta))$, then evidently $\operatorname{dist}(w(\theta), \partial G)>\operatorname{ch}(\theta)$ for some constant $c$, and since $\left(1-|z|^{2}\right)\left|\phi^{\prime}(z)\right| \geq \operatorname{dist}(\phi(z), \partial G)$, it follows that $\frac{1-|z(\theta)|^{2}}{1-|w(\theta)|^{2}}\left|\phi^{\prime}(z(\theta))\right| \geq \frac{c}{4}$, and so $C_{\phi}$ is not compact.

\section{CONCLUSION}

Although the conditions of Theorems 1 and 2 provide succinct analytic conditions on a function $\phi$ in order that it induce compact composition operators, it is desirable to have more geometric conditions. For example, it is clear from Section 3 that if $\phi$ is a conformal mapping which has only a finite number of nontangential cusps on the unit circle $\mathbb{T}$ and no other points of contact, then $C_{\phi}$ will be compact on $\mathscr{B}_{0}$. This raises the question of whether or not there is a $\phi \in \mathscr{B}_{0}$ such that $\overline{\phi(\mathbb{D})} \cap \mathbb{T}$ is infinite and $C_{\phi}$ is compact on $\mathscr{B}_{0}$. In this regard, it is known that if $\phi$ has nontangential limit of modulus one on a set of positive measure, then $\phi$ has an angular derivative at some point and so $C_{\phi}$ is not compact [Sh, p. 71]. Further information about compact operators considered from a geometric point of view, especially on $H^{2}$, can be found in [Sh] and [SSS].

Finally, if $\phi \in \mathscr{B}_{0}$ and $C_{\phi}$ is compact, then $\log \frac{1}{1-\bar{w} \phi(z)} \in \mathscr{B}_{0}$ for all $w \in \mathbb{T}$. Is the converse of this true?

\section{REFERENCES}

[A] Lars V. Ahlfors, Conformal invariants, McGraw-Hill, New York, 1973.

[ACP] J. M. Anderson, J. Clunie and Ch. Pommerenke, On Bloch functions and normal functions, J. Reine Angew. Math. 270 (1974), 12-37.

[D] Joseph Diestel, Sequences and series in Banach spaces, Springer-Verlag, New York, 1984. 
[G] John Garnett, Applications of harmonic measure, Univ. Arkansas Lecture Notes in the Math. Sci., vol. 8, John Wiley \& Sons, New York, 1986.

[P] Ch. Pommerenke, Boundary behaviour of conformal maps, Springer-Verlag, Berlin, 1992.

[RR1] R. Rochberg, Decomposition theorems for Bergman spaces and their applications, Operators and Function Theory (S. C. Power, ed.), Reidel, Dordrecht, 1985, pp. 225-277.

[RR2] R. Rochberg, Interpolation by functions in the Bergman spaces, Michigan Math. J. 29 (1982), 229-236.

[Sh] J. Shapiro, Composition operators and classical function theory, Springer-Verlag, New York, 1993.

[SSS] J. H. Shapiro, W. Smith, and D. A. Stegenga, Geometric models and compactness of composition operators, J. Funct. Anal. (to appear).

Department of Mathematics, Northwestern University, Evanston, IllinOIs 60208

E-mail address: madigan@math.nwu.edu

Department of Mathematics, Lamar University, Beaumont, TeXas 77710

E-mail address: matheson@math. lamar.edu 